\title{
Special issue on advances in learning classifier systems
}

\author{
Daniele Loiacono $\cdot$ Albert Orriols-Puig • \\ Ryan Urbanowicz
}

Published online: 22 May 2012

(C) Springer-Verlag 2012

Learning Classifier Systems (LCS) constitute a uniquely adaptable class of learning framework existing at the intersection of machine learning and evolutionary computation. Fundamentally, an LCS combines genetic search with an appropriate learning strategy to evolve a rule set which collectively describes a temporal or spatial problem. Since their conceptualization, a variety of algorithmic architectures and mechanisms have been introduced with the objective of improved functionality or application to new problem domains. As technology advances, the power and versatility of LCS algorithms makes them increasingly appealing.

Since 1992, the International Workshop on Learning Classifier Systems (IWLCS) has sought to promote the ongoing development of LCS architecture, application, and theory. Beginning in 2003, the IWLCS has been held annually, in association with the Genetic and Evolutionary Computation Conference (GECCO). This special issue includes original works as well as selected and revised papers from the 13th and 14th IWLCS, held in Portland, USA, during GECCO 2010, and Dublin, IR, during GECCO 2011.

\footnotetext{
D. Loiacono

Politecnico di Milano, P.zza Leonardo Da Vinci,

32, 20133 Milan, Italy

e-mail: loiacono@elet.polimi.it

A. Orriols-Puig

Google Inc, New York, NY, USA

e-mail: aorriols@gmail.com

R. Urbanowicz $(\bowtie)$

Department of Genetics, Dartmouth College,

Hanover, NH, USA

e-mail: Ryanurbanowicz@gmail.com
}

Over the last few years, there has been tremendous progress in (1) the systematic design of novel LCSs that can deal with additional problem topologies, (2) theoretical analyses incorporated to better understand and improve existing systems and (3) applications within important domains. The works collected in this special issue extend this trend, illustrating the maturity of LCSs and their applicability to hard real-world problems that currently elude solution.

In Production System Rules as Protein Complexes from Genetic Regulatory Networks: An Initial Study, Bull presents a new type of production system rules, by introducing a new, indirect encoding that views rules as protein complexes produced by the temporal behavior of an artificial genetic regulatory network. The experimental analysis shows that the new innovative approach has potential, and opens up different lines for future research.

Taking a look back to the origins of LCSs, in Risk Neutrality in Learning Classifier Systems Smith develops a novel model of risk-neutral reinforcement learning in a traditional Bucket Brigade credit-allocation market which is adjusted by a genetic algorithm. The work revises two important aspects in LCSs: default hierarchies and long chains of coupled classifiers. The new model is extensively discussed, reviving some ideas present in the first LCSs proposals by Holland.

In Analysing BioHEL Using Challenging Boolean Functions, the issue moves to different systematic analysis of current LCSs that enable a better understanding of the systems and give light to future improvements. In this paper, Franco et al. examine how BioHEL, a hierarchical evolutionary learning system specifically designed to cope with large-scale datasets, behaves on a family of Boolean functions whose complexity is varied along different dimensions. The experimental analysis shows the 
relationship between the learning time and different parameters used to represent problem complexity. Additionally, the study serves to identify some weaknesses in the fitness function and to point out different approaches to alleviate these problems, which will be investigated in further work.

In Function Approximation with LWPR and XCSF: A Comparative Study, Stalph et al. focus on XCSF, a variant of XCS for function approximation that has recently received a large amount of attention. Having identified the challenge of dealing with problems that have to be approximated from instances that are observed from an online stream, the paper compares XCSF to another stateof-the-art non-parametric regression algorithm capable of approximating multi-dimensional, non-linear functions online. The analysis systematically explores the trade-off between accuracy and population size, highlights the power of the evolutionary structuring capability of XCSF, and concludes with a broad discussion on the behavior of both systems on these type of learning problems.

In XCSF with Local Deletion: Preventing Detrimental Forgetting, Butz and Sigaud continue the efforts toward a better understanding of XCSF by studying its current global-population-based deleting scheme. This global scheme is shown to cause population instability and loss of performance on problems with specific complexities, specifically on problem spaces that are sampled non-uniformly and non-independently. This observation leads to the design of a new local deletion operator that yields more stable and accurate function approximations.
In On Principal Component Analysis for High-dimensional XCSR, Behdad et al. turn their attention to XCSR, a version of XCS that uses an interval-based representation. XCSR needs bigger populations and larger learning times as the size of the problem-and in particular, the number of attributes-increases. In order to alleviate the amount of resources needed by XCSR in these types of problems, the current work uses PCXCSR, a combination of PCA and $\mathrm{XCS}$ that aims at reducing the input space as well as the computational resources to explore it. The experimental analysis shows that PCXCSR significantly reduces the computational resources required to solve three large problems while maintaining accuracies similar to those observed with the original XCS.

Finally, in On XCSR for Electronic Fraud Detection Behdad et al. move to the application arena by applying XCSR to a hot real-world problem: fraud detection. The general characteristics of fraud detection are described and an abstract problem that exhibits these characteristics is defined. XCSR is applied to both artificial and real-world fraud-detection problems and a systematic analysis focused on different problem difficulties identified as characteristic of the fraud detection problem is conducted.

We are pleased to assemble this most recent collection of work in hopes that it will be of use to researchers interested in learning classifier systems. Our aim is to encourage further development and application of LCS within academia, business, and industry. We thank all authors that contributed to this volume as well as to the reviewers for their valuable insight. 